\title{
Theory of the Effect of Drag on the Orbital Inclination of an Earth Satellite
}

\author{
John P. Vinti
}

\begin{abstract}
The rotation of the earth's atmosphere with the earth gives rise to a change in the inclination of the orbit of an earth satellite. In this paper it is assumed that the drag is in the direction of the air velocity relative to the satellite and that its magnitude diminishes so rapidly with altitude that it is appreciable only near perigee in an elliptic orbit. Without further assumptions about the properties of the drag function, results are then deduced for the secular changes in inclination up to, but not including, the final ballistic stage of motion.
\end{abstract}

\section{Introduction}

Although the drag on an earth satellite is not well known, it is possible to express the secular changes that it produces in the inclination of the orbit, in terms of easily measurable quantities, provided only that the drag is given by a certain very general law. The present paper, which was suggested by a note by Wildhack, ${ }^{1}$ gives a more complete treatment of the problem, with more liberal use of mathematical methods.

\section{Forces and Frame of Reference}

If we adopt a Cartesian reference system with origin at the center of the earth and axis directions fixed relative to the fixed stars, the following forces act on a satellite:

$\mathbf{F}_{e}=$ inverse square gravitational force of the earth,

$\mathbf{F}_{0}=$ correction to $\mathbf{F}_{e}$, arising from oblateness of the earth,

$\mathbf{F}_{s}=$ gravitational force of the sun,

$\mathbf{F}_{m}=$ gravitational force of the moon;

$\mathbf{F}_{i s}=$ inertial force arising from acceleration of the earth, produced by the sun,

$\mathbf{F}_{i m}=$ inertial force arising from acceleration of the earth, produced by the moon,

$\mathbf{F}_{d}=$ drag force.

There are other forces, all of which may be disregarded as extremely small. Moreover, $\mathbf{F}_{s}+\mathbf{F}_{i s}$ and $\mathbf{F}_{m}+\mathbf{F}_{i m}$ are small compared to $\mathbf{F}_{o}$. We are thus left with the forces $\mathbf{F}_{e}$, $\mathbf{F}_{o}$, and $\mathbf{F}_{d}$.

The oblateness force $\mathbf{F}_{o}$ has a secular effect on the orbit, inasmuch as it produces a secular precession of the line of nodes and of the line of apsides. In considering changes in the inclination of the orbit, however, we are concerned with nutation and the effect of oblateness is only to produce a small oscillatory nutation. We shall therefore be concerned only with the forces $\mathbf{F}_{e}$ and $\mathbf{F}_{d}$, up to that point where we find results dependent on the (oblateness-induced) motion of the perigee. At that point it will then be sufficient to quote results by Garfinkel ${ }^{2}$ to find how oblateness influences the secular effect of drag on the inclination.

\section{Fundamental Theory}

With the above choice of reference system the center of the earth plays the role of a fixed point and the angular momentum $\mathbf{p}$ of the satellite is given by

$$
\mathbf{p}=m \mathbf{r} \times \dot{\mathbf{r}} .
$$

Here $\mathrm{m}$ is the mass of the satellite, $\mathbf{r}$ is the radius vector to it from the center of the earth, and $\mathbf{r}$ is the velocity of the satellite.

\footnotetext{
1 W. A. Wildhack, Science, 128, 309 (Aug. 8, 1958)

2 B. Garfinkel, Astron. J. 63, 88 (March 1958).
} 
As an orthonormal vector basis we choose $\mathbf{k}$ pointing northward along the axis of rotation of the earth, $\mathbf{i}$ pointing in such a fixed direction that at time $t=0$ its extension pierces the Greenwich meridian, and $\mathbf{j}$ so that $\mathbf{j}=\mathbf{k} \times \mathbf{i}$.

The orbit is a space curve, but it is natural to define the instantaneous plane of the orbit as the plane of two successive radius vectors, i. e., as the plane of $\mathbf{r}$ and $\mathbf{r}$ or the plane perpendicular to p. It is then convenient to define the directed unit normal to the orbit as a unit vector $\mathbf{1}_{p}$ along $\mathbf{p}$. The sense of the motion of the satellite relative to $\mathbf{1}_{p}$ is then given by the right-hand screw rule. We then define the inclination $\theta$ of the orbit as the angle between $\mathbf{k}$ and $\mathbf{1}_{p}$. (In terms of observable quantities $\theta$ is thus the greatest latitude reached by the satellite in a revolution.) Then

where $p \equiv|\mathbf{p}|$ and

$$
\mathbf{p}=p \mathbf{1}_{p},
$$

$$
\mathbf{1}_{p}=\mathbf{i} \sin \theta \cos \phi+\mathbf{j} \sin \theta \sin \phi+\mathbf{k} \cos \theta
$$

the angle $\phi$ being the angle between $\mathbf{i}$ and the projection of $\mathbf{p}$ on the equatorial plane $\{\mathbf{i}, \mathbf{j}\}$. Then $p, \theta$, and $\phi$ are the polar coordinates of $\mathbf{p}$.

The vector

$$
\mathbf{1}_{N}=\mathbf{k} \times \mathbf{1}_{p} \csc \theta
$$

is a unit vector lying both in the equatorial plane and in the plane of the orbit, so that it points along their intersection, the line of nodes. If $\mathbf{l}_{r}$ is a unit vector along $\mathbf{r}$, it follows from (1) that $\mathbf{1}_{r}$ is perpendicular to $\mathbf{1}_{p}$. Moreover $\mathbf{1}_{N}$ is perpendicular to $\mathbf{1}_{p}$, by (4), and so is $\mathbf{1}_{p} \times \mathbf{1}_{N}$. Thus the unit vectors $\mathbf{1}_{r}, \mathbf{1}_{N}$, and $\mathbf{1}_{p} \times \mathbf{1}_{N}$ are coplanar, since they are all perpendicular to $\mathbf{1}_{p}$. If now $\psi$ is the angle between the radius vector and the line of nodes, so that

we have

$$
\psi=\left(\mathbf{1}_{N}, \mathbf{1}_{r}\right),
$$

$$
\mathbf{1}_{r}=\mathbf{1}_{N} \cos \psi+\mathbf{1}_{p} \times \mathbf{1}_{N} \sin \psi,
$$

where the positive sense for increase of $\psi$ is from $\mathbf{1}_{N}$ towards $\mathbf{1}_{p} \times \mathbf{1}_{N}$.

From (3) and (4)

$$
\begin{aligned}
\mathbf{1}_{N} & =-\mathbf{i} \sin \phi+\mathbf{j} \cos \phi \\
\mathbf{k} \times \mathbf{1}_{N} & =-\mathbf{i} \cos \phi-\mathbf{j} \sin \phi .
\end{aligned}
$$

Comparison of (8) with (3) shows that

$$
\mathbf{1}_{p}=\mathbf{k} \cos \theta-\mathbf{k} \times \mathbf{1}_{N} \sin \theta
$$

By (4) it follows that $\mathbf{1}_{N}$ is perpendicular to $\mathbf{k}$, so that $\mathbf{k}, \mathbf{1}_{N}$, and $\mathbf{k} \times \mathbf{1}_{N}$ form another righthanded orthonormal basis, which turns out to be useful.

\section{Changes of Angular Momentum}

If $\mathbf{F}$ is the total force acting on the satellite, then

$$
\dot{\mathbf{p}}=\mathbf{r} \times \mathbf{F} .
$$

The secular changes with which we are here concerned are produced only by $\mathbf{F}_{d}$, so that for our present purposes

$$
\dot{\mathbf{p}}=\mathbf{r} \times \mathbf{F}_{d} .
$$

To find the rates of change of $p, \theta$, and $\phi$, it is now convenient to express each side of (11) in terms of the basis $\mathbf{k}, \mathbf{l}_{N}$, and $\mathbf{k} \times \mathbf{l}_{N}$. 
The left side may be so expressed by means of (2), (3), (7), (8), and (9), with the result

$$
\dot{\mathbf{p}}=\mathbf{k}(p \cos \theta-p \sin \theta) \dot{\theta}+\mathbf{l}_{N} p \sin \theta \dot{\phi}+\mathbf{k} \times \mathbf{l}_{N}(-\dot{p} \sin \theta-p \cos \theta \dot{\theta}) .
$$

\section{Drag Force and Drag Torque}

We assume the atmosphere of the earth to be rotating with it like a rigid body. If $\omega_{e}$ is the angular speed of rotation of the earth, the velocity w of the atmosphere (w for "wind"), relative to the inertial system $\mathbf{i}, \mathbf{j}, \mathbf{k}$, is

$$
\mathbf{w}=\omega_{e} \mathbf{k} \times \mathbf{r} .
$$

The velocity of the satellite relative to the inertial system is $\dot{\mathbf{r}}$. Its velocity $\mathbf{v}_{a}$ relative to the atmosphere is then

$$
\mathbf{v}_{a}=\dot{\mathbf{r}}-\mathbf{w}
$$

We assume the drag force to be in the direction of $-\mathbf{v}_{a}$, with magnitude dependent on $\mathbf{r}$ and the relative speed $|\dot{\mathbf{r}}-\mathbf{w}|$. Thus

$$
\mathbf{F}_{d}=f(r,|\dot{\mathbf{r}}-\mathbf{w}|)(\mathbf{w}-\dot{\mathbf{r}}) .
$$

Equation (15) is the "very general law" mentioned in the Introduction. If the drag is quadratic in the air speed $|\dot{\mathbf{r}}-\mathbf{w}|$, then the function $f$ is proportional to the air speed. No such special assumption is needed, however, for the purposes of this paper.

The drag torque is now given by

$$
\mathbf{r} \times \mathbf{F}_{d}=f \mathbf{r} \times \mathbf{w}-f \mathbf{r} \times \mathbf{r} .
$$

Now by (1)

$$
\mathbf{r} \times \dot{\mathbf{r}}=\mathbf{p} / m
$$

by $(13)$

$$
\mathbf{r} \times \mathbf{w}=\omega_{e} r^{2}\left[\mathbf{k}-\mathbf{l}_{r}\left(\mathbf{l}_{r} \cdot \mathbf{k}\right)\right],
$$

and by (6) and (9)

$$
\mathbf{l}_{r}=\mathbf{k} \sin \theta \sin \psi+\mathbf{l}_{N} \cos \psi+\mathbf{k} \times \mathbf{l}_{N} \cos \theta \sin \psi .
$$

From (16) through (19) it then follows that

$\mathbf{r} \times \mathbf{F}_{d}=-f \mathbf{p} / m+f \omega_{e} r^{2}\left[\mathbf{k}\left(1-\sin ^{2} \theta \sin ^{2} \psi\right)-\mathbf{1}_{N} \sin \theta \sin \psi \cos \psi-\mathbf{k} \times \mathbf{1}_{N} \sin \theta \cos \theta \sin ^{2} \psi\right]$.

This is the expression for the drag torque.

\section{Basic Equations}

On inserting (12) and (20) into (10) and equating corresponding coefficients of the basis vectors $\mathbf{k}, \mathbf{l}_{N}$, and $\mathbf{k} \times \mathbf{l}_{N}$, we find

$$
\begin{gathered}
\dot{p} \cos \theta-p \sin \theta \dot{\theta}=-f m^{-1} p \cos \theta+f \omega_{e} r^{2}\left(1-\sin ^{2} \theta \sin ^{2} \psi\right) \\
\dot{p} \sin \theta+p \cos \theta \dot{\theta}=-f m^{-1} p \sin \theta+f \omega_{e} r^{2} \sin \theta \cos \theta \sin ^{2} \psi \\
p \sin \theta \dot{\phi}=-f \omega_{e} r^{2} \sin \theta \sin \psi \cos \psi .
\end{gathered}
$$


Multiplication of (21.2) by $i=\sqrt{-1}$ and addition of the resulting equation to (21.1) gives, after division by $\exp i \theta$ :

$$
\dot{p}+i p \dot{\theta}=f m^{-1} p+f \omega_{e} r^{2}\left[\cos ^{2} \psi \exp (-i \theta)+\cos \theta \sin ^{2} \psi\right],
$$

from which there follow

$$
\begin{gathered}
\dot{p}=f m^{-1} p+f \omega_{e} r^{2} \cos \theta \\
p \dot{\theta}=-f \omega_{e} r^{2} \cos ^{2} \psi \sin \theta .
\end{gathered}
$$

Equations (23), together with (21.3), are the basic equations for the changes in $p$, $\theta$, and $\phi$ that arise from the drag.

\section{Stages of the Motion}

We assume that the motion of a satellite takes place in three stages:

Stage (1): The motion is that of a precessing and shrinking ellipse, with diminishing eccentricity, in which the elliptic elements are slowly varying functions of time. For our present purposes the relevant elliptic elements $l, e$, and $\psi_{p}$, along with the related quantities $p, r_{p}$, and $r_{a}$, may be chosen as follows:

$p=$ magnitude of the orbital angular momentum

$l=$ semi-latus rectum

$e=$ eccentricity

$r_{p}=$ radius at perigee

$r_{a}=$ radius at apogee

$\psi_{p}=$ value of $\psi$ at perigee.

The elliptic orbit is then characterized by the equation

$$
r=l\left[1+e \cos \left(\psi-\psi_{p}\right)\right]^{-1},
$$

where

$$
l=\left(G M m^{2}\right)^{-1} p^{2}
$$

and

$$
p=m r^{2} \dot{\psi},
$$

$M$ being the mass of the earth. From (24) it follows that

$$
\begin{aligned}
& r_{p}=l(1+e)^{-1} \\
& r_{a}=l(1-e)^{-1}
\end{aligned}
$$

from which

$$
\begin{aligned}
& l=2 r_{p} r_{a}\left(r_{a}+r_{p}\right)^{-1} \\
& e=\left(r_{a}-r_{p}\right)\left(r_{a}+r_{p}\right)^{-1} .
\end{aligned}
$$

Stage (2): A quasi-steady spiral stage, in which there are no apsides, so that $\dot{r}$ is small and negative throughout the stage, and in which the speed $v$ is given approximately by the formula for a circular orbit without drag. With neglect of oblateness this relation is

$$
v^{2}=G M / r .
$$


Stage (3): A final "ballistic" stage, which consists of a rapidly diminishing spiral, and in which $v$ is not given approximately by the formula (30) for a circular orbit. Quantitative treatment of such a final stage would require an accurate knowledge of the drag function, so that we omit consideration of it in this paper, restricting our attention to the changes in inclination that may occur in the first two stages.

We let $r_{p 0}$ and $r_{a 0}$ be the initial perigee and apogee radii in stage (1) and $r_{1}$ their final common value at the end of stage (1). The quantity $r_{1}$ is then the initial radius in stage (2), which we take to have a final radius $r_{2}$. In any actual case the quantities $r_{p 0}$ and $r_{a 0}$ would be given as initial conditions, while the radii $r_{1}$ and $r_{2}$ would have to be determined by observation-or calculated with use of an accurate expression for the drag. For rough estimates of the order of magnitude of the expected changes in inclination we shall here simply postulate arbitrary values of $r_{p 0}$ and $r_{a 0}$ and try to guess plausible values of $r_{1}$ and $r_{2}$.

\section{A Simplification}

Oblateness is known to produce no secular change in the inclination $\theta$, but only an oscillation with amplitude less than $0.05^{\circ}$. Suppose we also anticipate a result of the present paper, viz, that drag produces only a small secular change in $\theta$, of the order $0.3^{\circ}$. For a polar orbit, with $\theta_{0}=90^{\circ}$, we can then neglect the term in (23.1) that involves $\cos \theta$.

For any other orbit this term, although nonvanishing, is still small in comparison with the term $\mathrm{fm}^{-1} \mathrm{p}$. To show this, note that the ratio $R$ of the two terms satisfies

$$
R<m \omega_{e} r^{2} / p
$$

With use of (26), it follows that

$$
R<\omega_{e} \mid \dot{\psi}(\approx 1 / 15) \text {. }
$$

If we neglect this term we need consider only the simplified system

$$
\begin{gathered}
\dot{p}=-f m^{-1} p \\
p \dot{\theta}=-f \omega_{e} r^{2} \cos ^{2} \psi \sin \theta .
\end{gathered}
$$

\section{Stage (2): Quasi-Steady Spiral Orbit}

It is convenient to treat stage (2) first. In this stage we shall assume that the change in $r$ in one revolution is so small that we can neglect the corresponding change in $f$ compared to that in $\psi$. Presumably $f$ is proportional to the density, which in turn presumably varies like $K \exp \left[-\left(r-r_{e}\right) / s\right]$, where $r_{e}=$ mean earth radius= radius of sphere of same volume as the earth $=3,959$ miles and where $s$ is of the order 10 miles. Our neglect of the variation in $f$ in one revolution is thus equivalent to assuming that the corresponding decrease in $r$ is somewhat less than one mile. (Clearly it would not do to make such an assumption for stage (3), when $r$ may be diminishing much more rapidly.)

To obtain the secular change, we can then "secularize" the right side of (23.2) by replacing $\cos ^{2} \psi$ by $1 / 2$, thereby obtaining,

$$
p \dot{\theta}=-\frac{1}{2} f \omega_{e} r^{2} \sin \theta
$$

Division of (33) by (23.1a) then gives

$$
d \theta / d p=\frac{1}{2} m \omega_{e} r^{2} p^{-2} \sin \theta .
$$


Note that the unknown drag function has now dropped out from the theory - in any explicit form. In stage (2)

$$
v=r \dot{\psi},
$$

closely enough, so that

$$
p=m r v,
$$

by (26) and (35). Equations (30) and (36) then yield

$$
p=m(G M)^{1 / 2} r^{1 / 2} .
$$

For convenience, we now define $g_{e}$ by

$$
\mu \equiv G M=g_{e} r_{e}^{2}
$$

where $r_{e}=3959$ miles, the above mean radius of the earth, and where $g_{e}$ is a corresponding effective value of the acceleration of gravity at sea level, free from centrifugal effects. Here

$$
\begin{gathered}
g_{e}=32.224 \mathrm{ft} / \mathrm{sec}^{2} \\
\left(r_{e} / g_{e}\right)^{1 / 2}=805.4 \mathrm{sec} .
\end{gathered}
$$

Then

$$
p=m r_{e} g_{e}^{1 / 2} r^{1 / 2} .
$$

From (34) and (40) it follows for stage (2) that

$$
\csc \theta d \theta=0.25 \omega_{e} r_{e}^{-1} g_{e}^{-1 / 2} r^{1 / 2} d r
$$

and thus that

$$
\int_{\theta_{1}}^{\theta_{2}} \csc \theta d \theta=\ln \frac{\tan \left(\theta_{2} / 2\right)}{\tan \left(\theta_{1} / 2\right)}=\frac{\omega_{e}}{6}\left(r_{e} / g_{e}\right)^{1 / 2}\left[\left(r_{2} / r_{e}\right)^{3 / 2}-\left(r_{1} / r_{e}\right)^{3 / 2}\right]
$$

where $r_{1}$ and $\theta_{1}$ are the radius and the inclination of the orbit at the beginning of stage (2) and $r_{2}$ and $\theta_{2}$ are the corresponding quantities at the end of stage (2).

Ax Example: Suppose that the initial altitude in stage (2) is 200 miles and the final altitude 100 miles. Then

We find

$$
\begin{aligned}
& r_{1}=3959+200=4159 \text { miles } \\
& r_{2}=3959+100=4059 \text { miles } .
\end{aligned}
$$

$$
\left(r_{2} / r_{e}\right)^{3 / 2}-\left(r_{1} / r_{e}\right)^{3 / 2}=-0.03860 .
$$

Then since $\omega_{e}=2 \pi / 86,164 \mathrm{sec}^{-1}$, it follows from (42), (43.3), and (39.2) that

$$
\ln \frac{\tan \left(\theta_{2} / 2\right)}{\tan \left(\theta_{1} / 2\right)}=-0.0003781 .
$$

Thus $\theta_{2}$ does not differ much from $\theta_{1}$. If we now put

$$
\begin{gathered}
Q \equiv \frac{\omega_{e}}{6}\left(r_{e} / g_{e}\right)^{1 / 2}\left[\left(r_{1} / r_{e}\right)^{3 / 2}-\left(r_{2} / r_{e}\right)^{3 / 2}\right]>0 \\
\theta_{2} / 2 \equiv \eta \\
\theta_{1} / 2 \equiv \eta_{0}
\end{gathered}
$$


then, by (42)

$$
\tan \eta=\exp (-Q) \tan \eta_{0}
$$

To find from (46) a power series in $Q$ for

$$
\Delta \theta \equiv \theta_{2}-\theta_{1}
$$

that will be valid for all $\theta_{1}, \theta_{2}$ in the interval $(0, \pi)$, we use the fact that $\eta$ and $\eta_{0}$ are almost equal to each other, with each lying in the first quadrant, and proceed as follows. Multiply (46) by $i \cos \eta_{0}$ and add $\cos \eta_{0}$ to each side.

The result is

where

$$
\begin{aligned}
\cos \eta_{0} \sec \eta \exp i \eta & =\cos \eta_{0}+i\left(\sin \eta_{0}\right) \exp (-Q) \\
& =\left(\exp i \eta_{0}\right)\left[1-i \gamma \sin \eta_{0} \exp \left(-i \eta_{0}\right)\right]
\end{aligned}
$$

a small positive quantity. Then

$$
\gamma \equiv 1-\exp (-Q)
$$

$$
\begin{aligned}
\eta-\eta_{0} & =\operatorname{Im} \ln \left[1-i \gamma \sin \eta_{0} \exp \left(-i \eta_{0}\right)\right] \\
& =-\gamma \sin \eta_{0} \cos \eta_{0}-0.5 \gamma^{2} \sin 2 \eta_{0} \sin ^{2} \eta_{0}+\ldots,
\end{aligned}
$$

on expansion of the logarithm and selection of the imaginary part. Thus

$$
\Delta \theta \equiv 2\left(\eta-\eta_{0}\right)=-\gamma \sin \theta_{1}-\gamma^{2} \sin \theta_{1} \sin ^{2}\left(\theta_{1} / 2\right)+\ldots .
$$

With use of (48.3), this becomes

$$
\Delta \theta=-Q \sin \theta_{1}+Q^{2}\left[0.5-\sin ^{2}\left(\theta_{1} / 2\right)\right] \sin \theta_{1}+\ldots,
$$

which may be shortened, accurately enough, to

$$
\Delta \theta=-Q \sin \theta_{1}=-\frac{\omega_{e}}{6}\left(r_{e} / g_{e}\right)^{1 / 2}\left[\left(r_{1} / r_{e}\right)^{3 / 2}-\left(r_{2} / r_{e}\right)^{3 / 2}\right] \sin \theta_{1} .
$$

Then, since

$$
\frac{\omega_{e}}{6}\left(r_{e} / g_{e}\right)^{1 / 2}=0.009789 \text { radians }=0.5609^{\circ},
$$

we have, as our final result for stage (2):

$$
\Delta \theta=-0.5609\left[\left(r_{1} / r_{e}\right)^{3 / 2}-\left(r_{2} / r_{e}\right)^{3 / 2}\right] \sin \theta_{1} \text { degrees, }
$$

where $r_{e}=3959$ miles.

For the above example, where the altitude is assumed to diminish from 200 miles to 100 miles in stage (2), insertion into (55) of the numerical value 0.03860 from (43.3 gives the result

$$
\Delta \theta=-0.0217 \sin \theta_{1} \text { degrees. }
$$

This is a very small effect. We next consider stage (1), where we can often expect a larger effect, but where the calculations are more uncertain.

\section{Stage (1): Shrinking Elliptic Orbit}

In stage (1) the density and thus the function $f$ in (23.1a) and (23.2) are appreciable only when the satellite is very close to perigee. In effect $d \theta=0$ and $d p=0$ unless $r \approx r_{p}$. If we divide (23.2) by (23.1a), the result is

$$
\csc \theta d \theta=m \omega_{e} r^{2} p^{-2} \cos ^{2} \psi d p .
$$


In stage (2) we denote initial quantites by the subscript 0 and final quantities by the subscript 1. The integral of (57) over the whole of stage (1) is then

$$
\int_{\theta_{0}}^{\theta_{1}} \csc \theta d \theta=\ln \frac{\tan \left(\theta_{2} / 2\right)}{\tan \left(\theta_{0} / 2\right)}=m \omega_{e} \int_{p_{0}}^{p_{1}} r^{2} p^{-2} \cos ^{2} \psi d p
$$

In the light of the above remarks we may put

$$
r^{2} \cos ^{2} \psi=r_{p}^{2} \cos ^{2} \psi_{p}
$$

for any appreciable increment $d p$, with the result

$$
\ln \frac{\tan \left(\theta_{1} / 2\right)}{\tan \left(\theta_{0} / 2\right)}=m \omega_{e} \int_{p_{0}}^{p_{1}} r_{p}^{2} p^{-2} \cos ^{2} \psi_{p} d p
$$

Now the perigee radius changes little even in stage (1), so that we shall not be far wrong if we replace $r_{p}$ in (60) by either $r_{p 0}$ or $r_{1}$. Let us therefore replace $r_{p}$ by the arithmetic mean

$$
\overline{\boldsymbol{r}}=\frac{1}{2}\left(r_{p_{0}}+r_{1}\right) \text {. }
$$

In $(60) \psi_{p}$ is the angle between the line of nodes and the radius to perigee (the line of apsides). When oblateness is taken into account (but not drag), the rate of change of $\psi_{p}$ is given by Garfinkel (see footnote 2, eq. 53), whose result in the present notation is

$$
\dot{\psi}_{p}=3 \epsilon\left(r_{e} / l\right)^{2} n\left(1-1.25 \sin ^{2} \theta\right),
$$

where

$$
\epsilon \equiv\left(I_{p}-I_{t}\right) / M r_{e}^{2}=0.00109
$$

the quantities $I_{p}$ and $I_{t}$ being respectively the polar and transverse moments of inertia of the earth. We have here replaced Garfinkel's equatorial radius by our mean radius $r_{e}$, an unimportant change. The quantity $l$ is the semi-latus rectum, $\theta$ is the inclination of the orbit, and $n$ is the mean angular velocity of the satellite in its orbit; i. e.,

$$
n=\left(\mu / a^{3}\right)^{1 / 2},
$$

where the semi-major axis $a$ is given by

$$
a=l\left(1-e^{2}\right)^{-1}=\frac{1}{2}\left(r_{a}+r_{p}\right) .
$$

With the use of (64), (62), (38), and (39), we find

$$
\begin{aligned}
\dot{\psi}_{p} & =3 \epsilon\left(g_{e} / r_{e}\right)^{1 / 2}\left(r_{e} / a\right)^{7 / 2}\left(1-e^{2}\right)^{-2}\left(1-1.25 \sin ^{2} \theta\right) \\
& =20.1^{\circ}\left(r_{e} / a\right)^{7 / 2}\left(1-e^{2}\right)^{-2}\left(1-1.25 \sin ^{2} \theta\right) \text { per day. }
\end{aligned}
$$

As an example, suppose that $r_{p 0}=(3959+400)$ miles and $r_{a 0}=(3959+2000)$ miles and let us guess that $r_{p 1}=r_{a 1}=r_{1}=(3959+200)$ miles. (This latter figure corresponds to the beginning of stage (2), as assumed in our example above.) Then, by (64.2) and (29.2), $a_{0}=5159$ miles and $e_{0}=0.1552$, so that $e_{0}^{2}=0.0241$. Also $a_{1}=4159$ miles and $e_{1}=0$. The factor $\left(r_{e} / a\right)^{7 / 2}\left(1-e^{2}\right)^{-2}$ then increases from 0.415 to 0.842 , with a mean value of about 0.628 . Thus

$$
\left(\dot{\psi}_{p}\right)_{\text {mean }} \approx 12.6^{\circ}\left(1-1.25 \sin ^{2} \theta\right) \text { per day. }
$$

This amounts to $12.6^{\circ}$ per day for an equatorial orbit or $-3.15^{\circ}$ per day for a polar orbit.

Thus for a polar orbit the period of $\psi_{p}$ is about 3.7 months. If the lifetime of a polar satellite is less than this, it remains doubtful what to do about $\cos ^{2} \psi_{p}$ in (60), although even then we can establish an upper limit for $\left|\theta_{1}-\theta_{0}\right|$. 
If stage (1) lasts for a year or more, then $\psi_{p}$ goes through a number of cycles in this state, unless $\sin ^{2} \theta$ is too close to the value 0.8 , for which the inclination $\theta=63.4^{\circ}$. If we consider various possible orbits and let $\theta$ approach $63.4^{\circ}$, the period of $\psi_{p}$ becomes greater and greater, until at this critical inclination the angle $\psi_{p}$ would remain constant at its initial value during the whole of stage (1).

For durations of stage (1) longer than a year and unless $\theta \approx 63.4^{\circ}$, however, we may find the secular change in $\theta$ by replacing $\cos ^{2} \psi_{p}$ by $\frac{1}{2}$ in $(60)$, with the result

$$
\begin{aligned}
\ln \frac{\tan \left(\theta_{1} / 2\right)}{\tan \left(\theta_{0} / 2\right)} & =0.5 m \omega_{e} \bar{r}^{2}\left(p_{0}^{-1}-p_{1}^{-1}\right) \\
& =-0.5 \omega_{e}\left(r_{e} / g\right)^{1 / 2}\left(\bar{r} / r_{e}\right)^{2}\left[\left(r_{e} / r_{1}\right)^{1 / 2}-\left(r_{e} / l_{0}\right)^{1 / 2}\right],
\end{aligned}
$$

with the use of (25) and (38). Here

$$
l_{0}^{-1}=0.5\left(r_{a 0}^{-1}+r_{p 0}^{-1}\right)
$$

from (29.1).

We may evaluate $\theta_{1}-\theta_{0}$ from the logarithmic tangent expression, in the same manner that we found $\theta_{2}-\theta_{1}$ in stage (2). From (67) we find

and from (60) we find

$$
\theta_{1}-\theta_{0}=-0.5 L \quad\left(\theta \neq 63.4^{\circ}\right)
$$

$$
\theta_{1}-\theta_{0}=-L \cos ^{2} \psi_{p}\left(\theta \approx 63.4^{\circ} \text {, with } \psi_{p} \text { effectively constant }\right) .
$$

In (68) and (69)

In any case

$$
L \equiv \omega_{e}\left(r_{e} / g_{e}\right)^{1 / 2}\left(\bar{r} / r_{e}\right)^{2}\left[\left(r_{e} / r_{1}\right)^{1 / 2}-\left(r_{e} / l_{0}\right)^{1 / 2}\right]
$$

$$
\left|\theta_{1}-\theta_{0}\right|<L
$$

An Example: We consider the case where the initial perigee and apogee altitudes are respectively 400 miles and 2000 miles and assume that the final perigee and apogee altitudes of stage (1) are both 200 miles. The end of stage (1) in this example is then the same as the beginning of stage (2) in the previous example, so that the two examples together constitute one compound example. The $r_{p 0}=3959+400=4359$ miles, $r_{a 0}=3959+2000=5959$ miles, $r_{p 1}=r_{a 1}=$ $r_{1}=3959+200=4159$ miles, so that $L=0.346^{\circ}$. Then, by (68) through (71), we have for

Stage (1) (In miles, perigee $400 \rightarrow 200$; apogee $2000 \rightarrow 200$ )

$$
\begin{array}{ll}
\theta_{1}-\theta_{0}=-0.173^{\circ} \sin \theta_{0} & \left(\theta_{0} \not 63.4^{\circ}\right) \\
\theta_{1}-\theta_{0}=-0.346 \cos ^{2} \psi_{p} \sin \theta_{0} & \left(\theta_{0} \text { so close to } 63.4^{\circ} \text { that } \psi_{p} \text { is effectively constant. }\right)
\end{array}
$$

In any case

$$
\left|\theta_{1}-\theta_{0}\right|<0.346^{\circ} \sin \theta_{0}
$$

These results may be compared with those for

Stage (2) (In miles, altitude $200 \rightarrow 100$ )

$$
\theta_{2}-\theta_{1}=-0.022^{\circ} \sin \theta_{1} \approx-0.022^{\circ} \sin \theta_{0}
$$

The over-all results are that

$$
\begin{gathered}
\theta_{2}-\theta_{0}=-0.195^{\circ} \sin \theta_{0} \quad\left(\theta_{0} \neq 63.4^{\circ}\right) \\
\left|\theta_{2}-\theta_{0}\right|<0.368^{\circ} \sin \theta_{0}, \text { in any case. }
\end{gathered}
$$




\section{Summary}

The motion of an earth satellite relative to the earth's atmosphere produces a secular change in the inclination of the orbit. In predicting this change it is assumed that atmospheric drag acts in the direction of the air velocity relative to the satellite and that its magnitude depends on the relative air speed and in such a way on the altitude that it is appreciable only near the perigee, in the elliptic stage of motion. No other assumption is made about the drag.

If $\theta$ is the inclination of the orbit, $p$ the orbital angular momentum, $m$ the mass of the satellite, $\omega_{e}$ the angular speed of rotation of the earth, $r$ the distance of the satellite from the earth's center, and $\psi$ the angle between the radius vector and the line of nodes, it is then shown that $\csc \theta \mathrm{d} \theta=m \omega_{e} \mathrm{r}^{2} \cos ^{2} \psi d p$, to a good approximation, independently of the magnitude of the drag.

The motion is separated into an initial elliptic stage, a quasi-steady spiral stage, and a final ballistic stage. The secular change in $\theta$ is deduced for the spiral stage by integrating the above differential equation with use of an average value for $r$ and the average value $1 / 2$ for $\cos ^{2} \psi$. For the elliptic stage the secular change is found by putting $\mathrm{r}^{2} \cos ^{2} \psi$ equal to the perigee value $r_{p}^{2} \cos ^{2} \psi_{p}$ in the above equation and then using some results of Garfinkel (see footnote 2) concerning the motion of the perigee. Nothing can be said about the final ballistic stage, however, without accurate knowledge of the drag.

The secular change $\Delta \theta$ in the inclination, a quantity difficult to measure, is thus expressed, for the first two stages of motion, in terms of easily measurable satellite distances. As an example, it is assumed that a satellite, with initial perigee and apogee altitudes of 400 miles and 2,000 miles, falls to an altitude of 200 miles at the end of the elliptic stage and an altitude of 100 miles at the end of the quasi-steady spiral stage. If these data should happen to be correct, then $\Delta \theta$ would be $-0.022^{\circ} \sin \theta_{0}$ for the spiral stage and $-0.173^{\circ} \sin \theta_{0}$ for the elliptic stage. The latter figure depends on the assumption that $\theta_{0}$ is not close to the critical value $63.4^{\circ}$, for which there is no motion of the perigee relative to the line of nodes. In any case the total $|\Delta \theta|$ would always be less than $0.368^{\circ}$, for the assumed satellite altitudes, before the ballistic stage sets in. Once the ballistic stage begins, no predictions can be made by the methods of this paper.

Washington, September 15, 1958. 